\title{
Variación anual de las propiedades insecticidas de Peumus boldus sobre Sitophilus zeamais
}

\author{
Felipe Pérez(1), Gonzalo Silva(1), Maritza Tapia ${ }^{(1)}$ y Ruperto Hepp ${ }^{(1)}$
}

(1)Universidad de Concepción, Facultad de Agronomía, Dep. de Producción Vegetal, Vicente Méndez, № 595, Casilla 537 Chillán, Chile. E-mail: feperez@udec.cl, gosilva@udec.cl, martapia@udec.cl, rhepp@udec.cl

\begin{abstract}
Resumen - El objetivo del presente trabajo fue determinar la variación anual en las propiedades insecticidas de Peumus boldus Molina, en el control de Sitophilus zeamais Motschulsky, bajo condiciones de laboratorio. El polvo de hojas de $P$. boldus se evaluó durante 12 meses, en concentraciones de $0,5,1$ y $2 \%$ (p/p). Se evaluaron 36 tratamientos con tres repeticiones, en un diseño experimental completamente al azar, con un arreglo factorial. Se determinó el porcentaje de mortalidad y emergencia de insectos adultos e inmaduros y pérdida de peso y germinación del grano. La mayor mortalidad se obtuvo en los meses de agosto y septiembre de 2003, para las tres concentraciones con un $100 \%$, mientras que la menor fue en mayo de 2003 , cuando solo la concentración de $2 \%$ fue próxima a $100 \%$ de mortalidad. La menor emergencia de insectos adultos y pérdida de peso se obtuvieron en los mismos tratamientos. El efecto sobre estados inmaduros fue menor que contra adultos, y la germinación de los granos de maíz fue afectada por los polvos de P. boldus. Las propiedades insecticidas del polvo de hojas de $P$. boldus no son estables durante el año, Mayo es el mes con la menor eficacia insecticida, y la germinación de las semillas se ve afectada por el polvo.
\end{abstract}

Términos para indexación: gorgojo del maíz, granos almacenados, metabolitos secundarios.

\section{Annual variation of insecticide properties of Peumus boldus on Sitophilus zeamais}

\begin{abstract}
The objective of this work was to determine the annual variation of Peumus boldus Molina insecticidal properties for the control of Sitophilus zeamais Motschulsky, under laboratory conditions. The powder of $P$. boldus leaves was evaluated, during 12 months, in $0.5,1$, and $2 \%(\mathrm{w} / \mathrm{w})$ concentrations. Thirty-six treatments, with three replications, were evaluated in a completely randomized experimental design, with a factorial arrangement. Evaluations were made for adult and immature insect percentage mortality and emergence, grain weight loss and seed germination. The greatest mortality was obtained in August and September 2003, in the three concentrations with $100 \%$, and the lowest mortality was registered in May 2003, when only 2\% (w/w) reached almost $100 \%$ mortality. The lowest adult insect emergence and weight loss were obtained in the same treatments. The effect on immature stages was lower than on adults; and grain germination was affected by $P$. boldus powders. Insecticidal properties of $P$. boldus leaves powder are not stable during the year; May is the month with lesser insecticide efficacy; and the powder affects grain germination.
\end{abstract}

Index terms: maize weevil, stored grains, secondary metabolites.

\section{Introducción}

Las pérdidas de granos en el almacenaje es el principal problema que enfrenta el agricultor, después de la cosecha. La situación es especialmente importante en países en desarrollo, entre los productores a pequeña escala, quienes ven mermadas sus cosechas a causa de la destrucción de los granos, en los lugares de almacenaje, por roedores, insectos, hongos y bacterias (White, 1995).

En Chile, las plagas más importantes asociadas con los granos almacenados son el gorgojo del maíz
(Sitophilus zeamais Motschulsky; Coleoptera: Curculionidae), el gorgojo del arroz (Sitophilus oryzae Linnaeus; Coleoptera: Curculionidae) y la polilla de los cereales (Sitotroga cerealella Olivier; Lepidoptera: Gelechiidae) (Larraín, 1994). En el control de estas plagas se utilizan diversos agroquímicos, los cuales han derivado en el surgimiento de resistencia, acumulación de residuos en el ambiente, intoxicaciones y aumento de los costos de producción (White \& Leesch, 1996).

En la agricultura de subsistencia, el uso de extractos y polvos, obtenidos de plantas del mismo agroecosistema, constituye una opción muy útil para agricultores de 
escasos recursos, que poseen superficies de terreno muy pequeñas (Silva et al., 2001). El boldo (Peumus boldus Molina; Monimiaceae) es una especie prometedora, puesto que aplicada como polvo de hojas, tanto en laboratorio como bodega, ha obtenido niveles de control de S. zeamais superiores al 95\% (Silva et al., 2003a, 2003b, 2005).

Lamentablemente, los insecticidas de origen vegetal presentan variabilidad en cuanto al tipo y concentración de sus compuestos (Rodríguez et al., 2003), debido a factores como la época del año, ubicación geográfica y estado fenológico. De hecho, Bustamante (1999) indica que uno de los grandes problemas en el uso de compuestos de origen vegetal, como plaguicidas, es que en muchas oportunidades no se especifica la naturaleza del material a usar. Es decir, no se menciona en forma precisa la época de colecta, si son hojas jóvenes o senescentes, o frutos maduros o verdes, ya que se debe considerar que el contenido de los compuestos activos varía, dependiendo de la zona de producción, edad del material y época del año. Un ejemplo al respecto lo entregan Guan \& Bottrell (1994), quienes señalan que las inconsistencias que existen en la efectividad de neem (Azadirachta indica A. Juss), como controlador de plagas en campo, se deben a que las concentraciones de azadirachtina pueden diferir entre un lugar y otro. Sidhu et al. (2003) estudiaron los contenidos de azadirachtina A y B, de árboles de neem provenientes de 43 provincias de India, y encontraron diferencias inclusive entre árboles provenientes de la misma provincia y de agroecosistemas similares. En base a lo anterior, es lógico suponer que el polvo de $P$. boldus no está exento de esa problemática.

El objetivo del presente trabajo fue determinar la variación anual en las propiedades insecticidas de $P$. boldus, en el control de $S$. zeamais.

\section{Material y Métodos}

La recolección del follaje de $P$. boldus se realizó en un mismo árbol, en el parque de la Universidad de Concepción, en Chillán, Octava Región, Chile. Se efectuó en 12 fechas consecutivas, cada 30 días, del 30 de octubre de 2002 al 30 de septiembre de 2003. El criterio utilizado fue el propuesto por Vogel et al. (1997), que se basa en escoger hojas al azar, alrededor del árbol y en distintas posiciones dentro del mismo. Las hojas recolectadas fueron secadas en un horno de convección forzada, a $40^{\circ} \mathrm{C}$ por 48 horas, y se molieron con un molino eléctrico para granos de café.
Se utilizaron adultos de $S$. zeamais, los cuales provinieron de la colonia permanente del Laboratorio de Toxicología de Insecticidas, de la Facultad de Agronomía, de la Universidad de Concepción. Para obtener una cantidad suficiente de individuos, los insectos se reprodujeron en condiciones controladas de $25 \pm 2^{\circ} \mathrm{C}$ de temperatura, $70 \pm 5 \%$ de humedad relativa y fotofase de 16 horas.

Se utilizó la cultivar de maíz México, adquirido en el mercado de frutas y hortalizas de la ciudad de Chillán. Para asegurar que estuviera libre de insectos y residuos de insecticidas, se lavó y se puso en una cámara de refrigeración por 48 horas.

La metodología usada fue la propuesta por Lagunes \& Rodríguez (1989). Se colocaron $100 \mathrm{~g}$ de maíz en frascos de vidrio de $250 \mathrm{~mL}$, y se mezclaron con el polvo vegetal en concentraciones de $0,5,1$ y $2 \%(\mathrm{p} / \mathrm{p})$; cada frasco se infestó con 20 parejas de insectos de no más de 7 días de edad. La elección de las concentraciones se realizó según Lagunes (1994), quien indica que la concentración de polvo vegetal, para el control de plagas de granos almacenados, no debiera ser mayor al $1 \%$. Por este motivo se decidió evaluar, una concentración $100 \%$ menor $(0,5 \%)$ y otra $100 \%$ mayor $(2 \%)$ que esa concentración. La diferenciación de sexos se realizó usando el criterio de Halstead (1963), el que señala que el rostrum de la hembra es evidentemente más largo y menos ornamentado que el del macho. Cada frasco fue tapado con una tela de tul fijada con una banda elástica, rotulado con la información del respectivo tratamiento y ubicado en una cámara bioclimática, en las condiciones señaladas para la obtención masiva de individuos.

La mortalidad se evaluó 15 días después de la infestación. Se extrajeron todos los insectos y se cuantificaron los vivos y muertos para determinar el porcentaje de mortalidad, que fue corregido mediante la ecuación de Abbott (1925). Se consideró muerto a todo insecto que no se movió ante la aplicación de un estímulo físico, que fue el toque con una aguja de disección (FAO, 1980). Debido a las características del ciclo de S. zeamais, 55 días después de realizada la infestación, se evaluó el porcentaje de emergencia de insectos adultos (F1) y se consideró como $100 \%$ la emergencia del testigo. A los 55 días después de la infestación, también se contabilizó el número de granos dañados, y se calculó el porcentaje de pérdida de peso con la fórmula propuesta por Adams \& Schulten (1976). 
Para verificar si la fecha de recolección de las hojas tuvo alguna influencia en el poder de germinación de los granos mezclados con los polvos de P. boldus, se tomaron 30 semillas por cada tratamiento, incluido el testigo, y se colocaron a germinar en placas Petri acondicionadas con una toalla de papel húmeda durante siete días.

Para evaluar el efecto sobre estados inmaduros, frascos con $100 \mathrm{~g}$ de maíz cv. México se infestaron con 20 parejas de insectos, las cuales copularon por 15 días sin intervención. Posteriormente, se retiraron los insectos y se mezcló el grano con los polvos vegetales según el procedimiento mencionado. A los 55 días de realizada la infestación, se contabilizó la emergencia de la progenie (F1) que se comparó con un testigo absoluto.

El diseño experimental fue completamente al azar, con un arreglo factorial $3 \times 12$ (tres concentraciones $\mathrm{x}$ doce fechas de recolección del material vegetal). Los valores porcentuales fueron transformados a $\operatorname{arcsen}(\mathrm{x} / 100)^{0,5} \mathrm{y}$ se hizo un análisis de varianza y una comparación de medias, mediante una prueba de Tukey, al 5\% de probabilidad, con el programa SAS, versión 8.12 (SAS Institute, 1998).

\section{Resultados y Discusión}

La interacción concentración $\mathrm{x}$ fecha de recolección fue significativa para la mortalidad y emergencia de insectos adultos y para la pérdida de peso del grano $(\mathrm{p} \leq 0,05)$, pero no fue significativa para la germinación (Cuadro 1).

En la concentración $0,5 \%$, los valores más elevados de mortalidad, superior al 95\%, se obtuvieron en febrero, marzo, julio, agosto y septiembre de 2003, en tanto que la menor mortalidad (6,9\%) se obtuvo en mayo de 2003 (Cuadro 2). Con la sola excepción de mayo de 2003, donde se obtuvo un mínimo significativo de mortalidad para las concentraciones de 1 y $2 \%$, con un 47,3 y $92,9 \%$ respectivamente, en todas las restantes fechas de evaluación no se registró diferencia significativa $(p>0,05)$ entre ellas.

La concentración constituye un factor limitante puesto que sólo la concentración de $2 \%$ mostró, durante todo el período de evaluación, una toxicidad sobre el $90 \%$. Es decir, al aumentar la concentración también aumenta la mortalidad, lo que concuerda con los resultados de Silva et al. (2003a, 2005) quienes, en ambos casos, solamente con concentraciones de 1 y $2 \%$ de polvo de P. boldus, alcanzaron porcentajes de mortalidad de $S$. zeamais superiores al $90 \%$.

En octubre de 2002 y febrero, marzo, julio, agosto y septiembre de 2003, no hubo diferencia significativa ( $p>0,05)$ entre las tres concentraciones (Cuadro 2), mientras que en las fechas restantes se registró una mortalidad significativamente menor en la concentración de $0,5 \%$, lo que también ocurrió con la concentración de $1 \%$ en mayo de 2003. Por tanto, se genera una interacción concentración $\mathrm{x}$ fecha de recolección, lo que significa que dependiendo de la concentración usada y de la fecha de recolección del material vegetal, el porcentaje de mortalidad será mayor o menor. En consecuencia, se puede señalar que la peor fecha de recolección es mayo, cuando sólo la concentración de $2 \%$ alcanza niveles de mortalidad superiores al $90 \%$.

Considerándose la fenología de $P$. boldus, según Vogel et al. (2005a), y que aún no se realizan estudios para identificar compuestos en forma específica, mayo corresponde al término del receso vegetativo y principio de la floración, lo que implica que la concentración de compuestos en las hojas disminuye para concentrarse en la producción de flores, lo que podría explicar los menores valores de ese mes. Aunque, en cuanto a la concentración de los compuestos, Vogel et al. (1997, 1999, 2005b) señalan que la menor concentración de

Cuadro 1. Cuadrados medios y coeficiente de variación para mortalidad y emergencia de insectos y pérdida de peso y germinación de los granos $^{(1)}$.

\begin{tabular}{|c|c|c|c|c|c|}
\hline \multirow[t]{2}{*}{ Fuente de variación } & \multirow[t]{2}{*}{ Mortalidad } & \multicolumn{2}{|c|}{ Emergencia } & \multirow{2}{*}{$\begin{array}{c}\text { Pérdida de peso de los granos } \\
\text { EA }\end{array}$} & \multirow[t]{2}{*}{ Germinación de los granos } \\
\hline & & EA & EI & & \\
\hline Concentración & $1,93 *$ & $19,39 *$ & $7,66^{*}$ & $0,350 *$ & $0,93 *$ \\
\hline Fecha de recolección & $0,45^{*}$ & $0,19 *$ & $0,42 *$ & $0,010^{*}$ & $0,17^{*}$ \\
\hline $\begin{array}{l}\text { Concentración } \mathrm{x} \text { fecha de } \\
\text { recolección }\end{array}$ & $0,12 *$ & $0,09 *$ & $0,09 *$ & $0,010^{*}$ & 0,04 \\
\hline CV $(\%)$ & 9,51 & 17,21 & 21,70 & 25,50 & 16,60 \\
\hline
\end{tabular}

${ }^{(1)}$ EA: evaluación de adultos; EI: evaluación de inmaduros. *Significativo a $5 \%$ de probabilidad. 
compuestos activos, especialmente alcaloides y aceites esenciales, en el follaje de P. boldus, se produce en diciembre, lo que no coincide con la menor mortalidad de adultos de $S$. zeamais observada en mayo. Según los mismos autores, los valores máximos de compuestos se obtienen en julio, lo que se relaciona positivamente con la mortalidad, superior al $98 \%$, obtenida en ese mes con la concentración de $0,5 \%$, en el presente trabajo.

En el porcentaje de emergencia de insectos adultos, a la concentración de $0,5 \%$, no hubo diferencia significativa $(p>0,05)$ en casi todos los meses, a excepción nuevamente del mes de mayo de 2003, con un máximo significativo en el porcentaje de emergencia de un $98,2 \%$, valor que no registró diferencia significativa ( $p>0,05$ ) con el testigo (Cuadro 3).

La tendencia en el porcentaje de emergencia de adultos de $S$. zeamais con 1 y $2 \%$, durante los meses evaluados, se mantuvo en un $0 \%$ o con valores muy cercanos a este. Además, no hubo diferencias significativas $(p>0,05)$ para cada una de estas concentraciones, excepto en mayo de 2003 para $1 \%$ con un aumento significativo $(16,2 \%)$ del porcentaje de emergencia (Cuadro 3).

Estos resultados concuerdan con los de Páez et al. (1990) y Silva et al. (2003b, 2005), quienes obtuvieron emergencias de $S$. zeamais de 1,0 y $0,2 \%$ respectivamente, con una concentración de polvo de hojas de P. boldus de $1 \%$. Sin embargo, difiere de lo

Cuadro 2. Porcentaje de mortalidad de adultos de Sitophilus zeamais, en maíz tratado con polvo de hojas de Peumus boldus en concentraciones de $0,5,1$ y $2 \%$ (p/p), entre octubre de 2002 y septiembre de $2003^{(1)}$.

\begin{tabular}{lccc}
\hline Fecha de & \multicolumn{3}{c}{ Concentraciones $(\%)$} \\
\cline { 2 - 4 } recolección & 0,5 & 1 & 2 \\
\hline Octubre & $68,5 \mathrm{cdA}$ & $81,7 \mathrm{aA}$ & $100 \mathrm{aA}$ \\
Noviembre & $74,4 \mathrm{bcdB}$ & $100,0 \mathrm{aA}$ & $100 \mathrm{aA}$ \\
Diciembre & $50,8 \mathrm{~dB}$ & $96,6 \mathrm{aA}$ & $100 \mathrm{aA}$ \\
Enero & $60,7 \mathrm{~dB}$ & $100,0 \mathrm{aA}$ & $100 \mathrm{aA}$ \\
Febrero & $94,7 \mathrm{abcA}$ & $100,0 \mathrm{aA}$ & $100 \mathrm{aA}$ \\
Marzo & $98,3 \mathrm{abA}$ & $100,0 \mathrm{aA}$ & $100 \mathrm{aA}$ \\
Abril & $73,3 \mathrm{bcdB}$ & $100,0 \mathrm{aA}$ & $100 \mathrm{aA}$ \\
Mayo & $6,9 \mathrm{eB}$ & $47,3 \mathrm{bB}$ & $93 \mathrm{bA}$ \\
Junio & $50,9 \mathrm{~dB}$ & $92,9 \mathrm{aA}$ & $100 \mathrm{aA}$ \\
Julio & $98,3 \mathrm{abA}$ & $100,0 \mathrm{aA}$ & $100 \mathrm{aA}$ \\
Agosto & $100,0 \mathrm{aA}$ & $100,0 \mathrm{aA}$ & $100 \mathrm{aA}$ \\
Septiembre & $100,0 \mathrm{aA}$ & $100,0 \mathrm{aA}$ & $100 \mathrm{aA}$ \\
\hline
\end{tabular}

(1)Medias con letras mayúsculas diferentes entre columnas, para cada fecha, y letras minúsculas diferentes entre filas, para cada concentración, difieren entre si por la prueba de Tukey, a 5\% de probabilidad. reportado por Silva et al. (2003a), quienes obtuvieron una emergencia de $28,8 \%$ con polvo de hojas de $P$. boldus colectadas en octubre, valor que no coincide con lo obtenido en esta investigación $(0,9 \%)$ para el mismo mes.

El porcentaje de emergencia de insectos adultos se relaciona inversamente con la mortalidad, ya que los tratamientos que registraron menor porcentaje de mortalidad mostraron un mayor porcentaje de emergencia de insectos y viceversa. Solamente se registró diferencia significativa para porcentaje de emergencia, entre las tres concentraciones, en diciembre de 2002 y mayo y junio de 2003 (Cuadro 3). Las fluctuaciones en estos meses se explican por la interacción entre las variables estudiadas, relacionándose con lo obtenido para mortalidad de adultos; lo que confirma la concentración de $0,5 \%$ y la recolección de mayo como los resultados menos prometedores.

Los resultados de la pérdida de peso de granos infestados com $S$. zeamais también fueron influenciados por la interacción de los factores concentración $\mathrm{x}$ fecha de recolección, lo que explica la fluctuación de los valores porcentuales de los granos dañados, en las tres concentraciones, en el transcurso de los meses. Así, con la sola excepción de febrero de 2003, todas las concentraciones registraron diferencias significativas con el testigo ( $\mathrm{p} \leq 0,05)$ (Cuadro 4).

Los meses más relevantes, en cuanto a una menor pérdida de peso, fueron marzo y septiembre de 2003

Cuadro 3. Porcentaje de emergencia de adultos de Sitophilus zeamais, en maíz tratado con polvo de hojas de Peumus boldus en concentraciones de $0,5,1$ y $2 \%$ (p/p), entre octubre de 2002 y septiembre de $2003^{(1)}$.

\begin{tabular}{lcccc}
\hline Fecha de & \multicolumn{4}{c}{ Concentraciones $(\%)$} \\
\cline { 2 - 5 } recolección & Testigo & 0,5 & 1 & 2 \\
\hline Octubre & $100 \mathrm{aA}$ & $2,84 \mathrm{bcB}$ & $0,95 \mathrm{bB}$ & $0,00 \mathrm{aB}$ \\
Noviembre & $100 \mathrm{aA}$ & $4,18 \mathrm{bcB}$ & $1,32 \mathrm{bB}$ & $0,22 \mathrm{aB}$ \\
Diciembre & $100 \mathrm{aA}$ & $6,90 \mathrm{bcB}$ & $0,52 \mathrm{bC}$ & $0,17 \mathrm{aC}$ \\
Enero & $100 \mathrm{aA}$ & $16,90 \mathrm{bcB}$ & $2,20 \mathrm{bB}$ & $2,20 \mathrm{aB}$ \\
Febrero & $100 \mathrm{aA}$ & $0,62 \mathrm{cB}$ & $0,00 \mathrm{bB}$ & $0,00 \mathrm{aB}$ \\
Marzo & $100 \mathrm{aA}$ & $0,00 \mathrm{cB}$ & $0,00 \mathrm{bB}$ & $0,00 \mathrm{aB}$ \\
Abril & $100 \mathrm{aA}$ & $2,43 \mathrm{bcB}$ & $0,00 \mathrm{bB}$ & $0,00 \mathrm{aB}$ \\
Mayo & $100 \mathrm{aA}$ & $98,20 \mathrm{aA}$ & $16,20 \mathrm{aB}$ & $1,15 \mathrm{aB}$ \\
Junio & $100 \mathrm{aA}$ & $18,40 \mathrm{bB}$ & $0,21 \mathrm{bC}$ & $0,00 \mathrm{aC}$ \\
Julio & $100 \mathrm{aA}$ & $0,00 \mathrm{cB}$ & $0,00 \mathrm{bB}$ & $0,00 \mathrm{aB}$ \\
Agosto & $100 \mathrm{aA}$ & $2,45 \mathrm{bcB}$ & $0,49 \mathrm{bB}$ & $0,00 \mathrm{aB}$ \\
Septiembre & $100 \mathrm{Aa}$ & $1,47 \mathrm{bcB}$ & $1,47 \mathrm{bB}$ & $0,74 \mathrm{aB}$ \\
\hline
\end{tabular}

(1)Medias con letras mayúsculas diferentes entre columnas, para cada fecha, y letras minúsculas diferentes entre filas, para cada concentración, difieren entre si por la prueba de Tukey, a 5\% de probabilidad. 
con un $0 \%$ para las tres concentraciones, y julio de 2003 con $0 \%$ al 1 y $2 \%$ y $0,03 \%$ al $0,5 \%$. La pérdida de peso del grano fue baja en todos los meses y para las tres concentraciones, y difirió solamente en mayo de 2003 con $8,9 \%(0,5 \%)$ y $2,1 \%(1 \%)$, los cuales fueron los valores máximos obtenidos. Estos resultados se relacionan con los obtenidos en la mortalidad de adultos de acuerdo a la fecha de recolección, puesto que en mayo de 2003 se obtuvo la menor mortalidad de $S$. zeamais y el mayor porcentaje de granos dañados. Esos resultados son similares a los de Silva et al. (2003b), quienes observaron una pérdida de peso de sólo $0,09 \%$ al usar $1 \%$ de concentración de polvo de hojas de P. boldus, aunque no se especifica la fecha de recolección. Silva et al. (2003a), usaron 1 y $2 \%$ de polvo de follaje de $P$. boldus recolectado en octubre, y obtuvieron pérdidas de peso de 1,7 y $1,0 \%$, lo que no concuerda con el presente trabajo.

En la germinación, los granos tratados con polvo de hojas de P. boldus, en las distintas concentraciones, presentaron diferencias numéricas respecto al testigo, ya que al $0,5,1$ y $2 \%(\mathrm{p} / \mathrm{p})$ los valores fueron $64,9,56,9$ y $50,2 \%$, mientras que para el testigo fue de $81,9 \%$. Los resultados indican una reducción importante en el poder germinativo de las semillas al aumentar la concentración de polvo de hojas de P. boldus (Cuadro 5). En este análisis, a diferencia de los anteriores, no hubo interacción entre la fecha de recolección y la

Cuadro 4. Porcentaje de pérdida de peso de granos infestados con Sitophilus zeamais, tratados con polvo de hojas de Peumus boldus en concentraciones de $0,5,1$ y $2 \%(\mathrm{p} / \mathrm{p})$, entre octubre de 2002 y septiembre de $2003^{(1)}$.

\begin{tabular}{lllll}
\hline Fecha de & \multicolumn{4}{c}{ Concentraciones (\%) } \\
\cline { 2 - 5 } recolección & \multicolumn{1}{c}{ Testigo } & \multicolumn{1}{c}{0,5} & \multicolumn{1}{c}{1} & \multicolumn{1}{c}{2} \\
\hline Octubre & $2,8 \mathrm{efA}$ & $0,51 \mathrm{cdeB}$ & $0,32 \mathrm{bcdBC}$ & $0,02 \mathrm{bcC}$ \\
Noviembre & $5,6 \mathrm{bcdA}$ & $0,54 \mathrm{cdeB}$ & $0,12 \mathrm{cdBC}$ & $0,02 \mathrm{bcC}$ \\
Diciembre & $6,8 \mathrm{abcA}$ & $0,79 \mathrm{bcdeB}$ & $0,07 \mathrm{cdC}$ & $0,01 \mathrm{bcC}$ \\
Enero & $2,8 \mathrm{defA}$ & $1,31 \mathrm{bcB}$ & $0,40 \mathrm{bcC}$ & $0,60 \mathrm{aBC}$ \\
Febrero & $1,8 \mathrm{fA}$ & $1,29 \mathrm{bcdA}$ & $1,10 \mathrm{abA}$ & $0,80 \mathrm{aA}$ \\
Marzo & $7,3 \mathrm{abcA}$ & $0,00 \mathrm{eB}$ & $0,00 \mathrm{~dB}$ & $0,00 \mathrm{cB}$ \\
Abril & $8,9 \mathrm{abA}$ & $1,20 \mathrm{bcdeB}$ & $0,06 \mathrm{cdB}$ & $0,03 \mathrm{bcB}$ \\
Mayo & $7,3 \mathrm{abcA}$ & $8,90 \mathrm{aA}$ & $2,10 \mathrm{aB}$ & $0,12 \mathrm{bC}$ \\
Junio & $6,8 \mathrm{abcA}$ & $3,20 \mathrm{bB}$ & $0,05 \mathrm{cdC}$ & $0,00 \mathrm{cC}$ \\
Julio & $9,6 \mathrm{aA}$ & $0,03 \mathrm{deB}$ & $0,00 \mathrm{~dB}$ & $0,00 \mathrm{cB}$ \\
Agosto & $4,5 \mathrm{cdeA}$ & $0,40 \mathrm{cdeB}$ & $0,09 \mathrm{cdB}$ & $0,00 \mathrm{cB}$ \\
Septiembre & $5,2 \mathrm{cdeA}$ & $0,00 \mathrm{eB}$ & $0,00 \mathrm{~dB}$ & $0,00 \mathrm{cB}$ \\
\hline
\end{tabular}

${ }^{(1)}$ Medias con letras mayúsculas diferentes entre columnas, para cada fecha, y letras minúsculas diferentes entre filas, para cada concentración, difieren entre si por la prueba de Tukey, a 5\% de probabilidad. concentración. En noviembre de 2002, se registró un máximo de $77,5 \%$, significativamente diferente $(p \leq 0,05)$ de lo obtenido en marzo, abril, julio, agosto y septiembre de 2003. La concentración fue el factor determinante ya que aumentarla causa una mayor reducción en la germinación del maíz, tal vez debido a un efecto fitotóxico sobre la semilla. Sin embargo, esos resultados son congruentes a los obtenidos por Silva et al. (2003a, 2005), quienes a pesar de obtener una disminución en la germinación de los granos a medida que aumentaba la concentración, no detectaron diferencias significativas al comparar las concentraciones de 1 y $2 \%$.

El porcentaje de emergencia de insectos adultos, cuando se intentó controlar los estados inmaduros de S. zeamais, sólo en febrero, marzo, abril y julio de 2003 presentó diferencias significativas $(\mathrm{p} \leq 0,05)$ entre las concentraciones evaluadas; situación que no se dio en el resto de los meses (Cuadro 6). En febrero y marzo de 2003, las diferencias fueron significativas $(p \leq 0,05)$ en las tres concentraciones, mientras que en abril y julio de 2003 sólo se presentaron diferencias significativas $(\mathrm{p} \leq 0,05)$ en la concentración de $2 \%$ con valores de 20,6 y $4,3 \%$.

En el análisis para $0,5 \%$, no se registró diferencia significativa entre las fechas de recolección (Cuadro 6); para $1 \%$ se registró un valor máximo de $80,9 \%$, en noviembre de 2002 , el cual difirió $(p \leq 0,05)$ de agosto y septiembre de 2003, que mostraron los valores mínimos (8,4 y $19,9 \%)$. El máximo significativo de $67,9 \%$ para

Cuadro 5. Porcentaje de germinación de maíz, tratado con polvo de hojas de Peumus boldus en concentraciones de 0,5, 1 y $2 \%$ (p/p), para el control de adultos de Sitophilus zeamais, entre octubre de 2002 y septiembre de $2003^{(1)}$.

\begin{tabular}{lccccl}
\hline Fecha de & \multicolumn{4}{c}{ Concentraciones (\%) } & \multirow{2}{*}{ Promedio } \\
\cline { 2 - 5 } recolección & Testigo & $0,5 \%$ & $1 \%$ & $2 \%$ & \\
\hline Octubre & 80,0 & 73,3 & 66,6 & 63,3 & $70,80 \mathrm{abc}$ \\
Noviembre & 83,3 & 70,0 & 80,0 & 76,6 & $77,48 \mathrm{a}$ \\
Diciembre & 83,3 & 80,0 & 70,0 & 66,6 & $74,97 \mathrm{ab}$ \\
Enero & 83,3 & 73,3 & 66,6 & 66,6 & $72,45 \mathrm{abc}$ \\
Febrero & 76,6 & 73,3 & 66,6 & 53,3 & $67,45 \mathrm{abcde}$ \\
Marzo & 80,0 & 63,3 & 43,3 & 43,3 & $57,48 \mathrm{bcde}$ \\
Abril & 73,3 & 66,6 & 43,3 & 36,6 & $54,95 \mathrm{cde}$ \\
Mayo & 83,3 & 76,6 & 73,3 & 66,6 & $74,95 \mathrm{ab}$ \\
Junio & 83,3 & 80,0 & 60,0 & 46,6 & $67,48 \mathrm{abcd}$ \\
Julio & 86,6 & 46,6 & 33,3 & 30,0 & $49,13 \mathrm{de}$ \\
Agosto & 83,3 & 40,0 & 36,6 & 26,6 & $46,63 \mathrm{e}$ \\
Septiembre & 86,6 & 36,6 & 43,3 & 26,6 & $48,27 \mathrm{e}$ \\
\hline Promedio & $81,9 \mathrm{a}$ & $64,9 \mathrm{~b}$ & $56,9 \mathrm{bc}$ & $50,2 \mathrm{c}$ & \\
\hline
\end{tabular}

${ }^{(1)}$ Medias con letras diferentes en la columna difieren entre si por la prueba de Tukey, a 5\% de probabilidad. 
Cuadro 6. Porcentaje de emergencia de Sitophilus zeamais, en maíz tratado con de polvo de hojas de Peumus boldus en concentraciones de $0,5,1$ y $2 \%(\mathrm{p} / \mathrm{p})$, para el control de estados inmaduros, entre octubre de 2002 y septiembre de $2003^{(1)}$.

\begin{tabular}{lllll}
\hline Fecha de & \multicolumn{4}{c}{ Concentraciones $(\%)$} \\
\cline { 2 - 5 } recolección & Testigo & \multicolumn{1}{c}{0,5} & \multicolumn{1}{c}{1} \\
\hline Octubre & $100 \mathrm{aA}$ & $63,9 \mathrm{aB}$ & $49,4 \mathrm{abcB}$ & $47,6 \mathrm{abcB}$ \\
Noviembre & $100 \mathrm{aA}$ & $85,0 \mathrm{aA}$ & $80,9 \mathrm{aA}$ & $67,9 \mathrm{aA}$ \\
Diciembre & $100 \mathrm{aA}$ & $84,1 \mathrm{aAB}$ & $73,4 \mathrm{abAB}$ & $50,1 \mathrm{abcB}$ \\
Enero & $100 \mathrm{aA}$ & $42,9 \mathrm{aB}$ & $30,8 \mathrm{abcB}$ & $21,2 \mathrm{abcdeB}$ \\
Febrero & $100 \mathrm{aA}$ & $75,1 \mathrm{aB}$ & $44,7 \mathrm{abcC}$ & $12,6 \mathrm{bcdeD}$ \\
Marzo & $100 \mathrm{aA}$ & $89,6 \mathrm{aA}$ & $51,0 \mathrm{abcB}$ & $8,9 \mathrm{cdeC}$ \\
Abril & $100 \mathrm{aA}$ & $73,1 \mathrm{aB}$ & $30,7 \mathrm{abcBC}$ & $20,6 \mathrm{bcdeC}$ \\
Mayo & $100 \mathrm{aA}$ & $55,1 \mathrm{aB}$ & $25,7 \mathrm{abcB}$ & $38,1 \mathrm{abcdB}$ \\
Junio & $100 \mathrm{aA}$ & $80,5 \mathrm{aA}$ & $69,6 \mathrm{abcA}$ & $51,5 \mathrm{abA}$ \\
Julio & $100 \mathrm{aA}$ & $75,0 \mathrm{aB}$ & $42,1 \mathrm{abcB}$ & $4,3 \mathrm{deC}$ \\
Agosto & $100 \mathrm{aA}$ & $31,6 \mathrm{aB}$ & $8,4 \mathrm{cB}$ & $1,1 \mathrm{eB}$ \\
Septiembre & $100 \mathrm{aA}$ & $33,1 \mathrm{aB}$ & $19,9 \mathrm{bcB}$ & $2,9 \mathrm{eB}$ \\
\hline
\end{tabular}

${ }^{(1)}$ Medias con letras mayúsculas diferentes entre columnas, para cada fecha, y letras minúsculas diferentes entre filas, para cada concentración, difieren entre si por la prueba de Tukey, a $5 \%$ de probabilidad.

$2 \%$ se obtuvo en noviembre de 2002 , el cual fue diferente $(\mathrm{p} \leq 0,05)$ respecto a agosto y septiembre de 2003 , que tuvieron valores mínimos ( 1,1 y $2,9 \%)$.

Existe un efecto tóxico del polvo de $P$. boldus sobre estados inmaduros de $S$. zeamais, especialmente a la concentración mayor (2\%), que disminuyó en noviembre y diciembre de 2002 y junio de 2003 ya que propiciaron una emergencia superior al 50\% en relación al testigo.

\section{Conclusiones}

1. Las propiedades insecticidas del polvo de hojas de Peumus boldus no son estables durante el año.

2. Mayo es el mes con la menor eficacia insecticida del polvo de hojas de Peumus boldus.

3. El polvo de hojas de Peumus boldus afecta la germinación del maíz.

\section{Agradecimientos}

A la Dirección de Investigación de la Universidad de Concepción, por el apoyo financiero.

\section{Referencias}

ABBOTT, W.S. A method for computing the effectiveness of an insecticide. Journal of Economic Entomology, v.18, p.265-267, 1925.

ADAMS, J.M.; SCHULTEN, G.G.M. Losses caused by insects, mites and microorganisms. In: AMERICAN ASSOCIATION OF
CEREAL CHEMISTS. Postharvest grain loss assessment methods. Slough, 1976. p.83-93.

BUSTAMANTE, M. Plaguicidas botánicos; una mentira o una alternativa para el pequeño agricultor. In: SIMPOSIO NACIONAL SOBRE SUBSTANCIAS VEGETALES Y MINERALES EN EL COMBAte DE PlagAS, 5., 1999, Aguascalientes. Anais. Aguascalientes, 1999. p.61-70.

FAO. Recommended methods for measurement of pest resistance to pesticides. Rome, 1980. 132p. (FAO. Plant production and protection paper, 21).

GUAN, L.G.; BOTTRELL, D.G. Neem pesticides in rice: potential and limitations. Manila: Irri, 1994. 69p.

HALSTEAD, D.G.H. External sex differences in stored-products: Coleoptera. Bulletin of Entomological Research, v.54, p.119134, 1963.

LAGUNES, A. Extractos de polvos vegetales y polvos minerales para el combate de plagas del maíz y del frijol en la agricultura de subsistencia. México: Colegio de Postgraduados/Usaid/Conacyt/ Boruconsa, 1994. 35p.

LAGUNES, A.; RODRÍGUEZ, C. Búsqueda de la tecnología apropiada para el combate de plagas del maíz almacenado en condiciones rústicas. Montecillo: Conacyt/Colegio de Postgraduados, 1989. 150p.

LARRAÍN, P. Manejo integrado de plagas en granos almacenados. Investigación y Progreso Agropecuario, v.81, p.10-16, 1994.

PÁEZ, A.; LAGUNES, A.; CARRILLO, J.L.; RODRÍGUEZ, J.C. Polvos vegetales y minerales para el combate del gorgojo del maíz Sitophilus zeamais Motschulsky (Coleoptera: Curculionidae) en maíz almacenado. Agrociencia, v.1, p.35-46, 1990.

RODRÍGUEZ, C.; SILVA, G.; VENDRAMIM, J. Insecticidas de origen vegetal. In: SILVA, G.; HEPP, R. (Ed.). Bases para el manejo racional de insecticidas. Chillán: Universidad de Concepción/ Fundación para la Innovación Agraria, 2003. p.87-112.

SAS INSTITUTE (Cary, Estados Unidos). Languague guide for personal computers release 6.03. Cary, 1998. 1028p.

SIDHU, O.P.; KHUMAR, V.; BEHL, H.M. Variability in Neem (Azadirachta indica) with respect to azadirachtin content. Journal of Agricultural and Food Chemistry, v.51, p.910-915, 2003.

SILVA, G.; LAGUNES, A.; RODRÍGUEZ, J.C. Control de Sitophilus zeamais (Coleoptera: Curculionidae) con polvos vegetales solos y en mezcla con carbonato de calcio en maíz almacenado. Ciencia e Investigación Agraria, v.30, p.152-160, 2003b.

SILVA, G.; LAGUNES, A.; RODRÍGUEZ, J.C.; RODRÍGUEZ, D. Insecticidas vegetales; una vieja nueva opción en el manejo de insectos. Manejo Integrado de Plagas y Agroecología, v.66, p.4-12, 2001.

SILVA, G.; ORREGO, O.; HEPP, R.; TAPIA, M. Búsqueda de plantas con propiedades insecticidas para el control de Sitophilus zeamais en maíz almacenado. Pesquisa Agropecuária Brasileira, v.40, p.1117, 2005. 
SILVA, G.; PIZARRO, D.; CASALS, P.; BERTI, M. Evaluación de plantas medicinales en polvo para el control de Sitophilus zeamais Motschulsky en maíz almacenado. Revista Brasileira de Agrociência, v.9, p.383-388, 2003a.

VOGEL, H.; RAZMILIC, I.; ACEVEDO, P.; GONZÁLEZ, B. Alkaloid and essential oil concentration in different populations of Peumus boldus. Acta Horticulturae, v.676, p.181-184. 2005b.

VOGEL, H.; RAZMILIC, I.; DOLL, U. Contenido de aceite esencial $\mathrm{y}$ alcaloides en diferentes poblaciones de boldo (Peumus boldus Mol.). Ciencia e Investigación Agraria, v.24, p.1-6, 1997.

VOGEL, H.; RAZMILIC, I.; MUÑOZ, M.; DOLL, U.; SAN MARTÍN, J. Studies of genetic variation of essential oil and alkaloid content in Boldo (Peumus boldus). Planta Medica, v.65, p.90-91, 1999.

VOGEL, H.; RAZMILIC, I.; SAN MARTÍN, J.; DOLL, U.; GONZÁLEZ, B. Plantas medicinales chilenas: experiencias de domesticación y cultivo de Boldo, Matico, Bailahuén, Canelo, Peumo y Maqui. Talca: Editorial Universidad de Talca, 2005a. 192p.

WHITE, D.G. Insects, mites, and insecticides in stored-grain ecosystems. In: JAYAS, D.S.; WHITE, N.D.; MUIR, W. (Ed.). Stored grain-ecosystems. New York: M. Dekker, 1995. p.123-168.

WHITE, D.G.; LEESCH, J. Chemical control. In: SUBRAMANYAM, B.; HAGSTRUM, D. (Ed.). Insects in stored products. New York: M. Dekker, 1996. p.287-330.

Recibido el 13 de julio de 2006 e aceptado el 11 de abril de 2007 